\title{
REVIEW
}

\section{Clinical review: Clinical imaging of the sublingual microcirculation in the critically ill - where do we stand?}

\author{
Rick Bezemer ${ }^{1,2 *}$, Sebastiaan A Bartels 1,2,3, Jan Bakker' and Can Ince $e^{1,2}$
}

\begin{abstract}
A growing body of evidence exists associating depressed microcirculatory function and morbidity and mortality in a wide array of clinical scenarios. It has been suggested that volume replacement therapy using fluids and/or blood in combination with vasoactive agents to modulate macro- and microvascular perfusion might be essential for resuscitation of severely septic patients. Even after interventions effectively optimizing macrocirculatory hemodynamics, however, high mortality rates still persist in critically ill and especially in septic patients. Therefore, rather than limiting therapy to macrocirculatory targets alone, microcirculatory targets could be incorporated to potentially reduce mortality rates in these critically ill patients. In the present review we first provide a brief history of clinical imaging of the microcirculation and describe how microcirculatory imaging has been of prognostic value in intensive care patients. We then give an overview of therapies potentially improving the microcirculation in critically ill patients and propose a clinical trial aimed at demonstrating that therapy targeting improvement of the microcirculation results in improved organ function in patients with severe sepsis and septic shock. We end with some recent technological advances in clinical microcirculatory image acquisition and analysis.
\end{abstract}

\section{Background}

The microcirculation may have a key role in the development of (multiple) organ failure in the critically ill and the main aim of hemodynamic resuscitation in these

*Correspondence: r.bezemer@amc.uva.nl

2Department of Translational Physiology, Academic Medical Center, University of Amsterdam, Meibergdreef 9, 1105 AZ Amsterdam, the Netherlands

Full list of author information is available at the end of the article patients is to restore microcirculatory perfusion and tissue oxygenation to prevent organ hypoxia and maintain organ function [1-3]. It has been recognized that therapeutic interventions should be delivered as early as possible $[3,4]$ and early protocol-driven resuscitation strategies (for example, early goal-directed therapy) targeting global hemodynamic parameters have been associated with the best clinical outcome in randomized controlled clinical trials $[4,5]$. However, even after interventions effectively optimizing macrocirculatory hemodynamics (for example, cardiac filling pressure, cardiac output, blood pressure, and central or mixed venous oxygen saturation), high mortality rates still persist [6]. In this light, it has been shown that improvement of macrocirculatory hemodynamics does not guarantee (sufficient) improvement of the microcirculation [2].

In critical illness, and especially in sepsis and shock, microcirculatory dysfunction may arise as a result of several factors, such as endothelial dysfunction, leukocyte-endothelium interactions, coagulation and inflammatory disorders, hemorheological abnormalities, and a disturbed balance between oxygen delivery and oxygen consumption [7]. This microcirculatory dysfunction is characterized by heterogeneous abnormalities in blood flow with some capillaries being non- or hypo-perfused while others are normally or even hyper-perfused. Due to the dysregulated heterogeneous flow distribution, weak microcirculatory units may become hypoxic. This is the main reason why monitoring systemic hemodynamicderived and oxygen-derived variables is not able to sense such microcirculatory dysfunction. Therefore, rather than limiting (early) goal-directed therapy to macrocirculatory targets alone, microcirculatory targets could be incorporated to more effectively resuscitate the microcirculation and thereby potentially reduce mortality rates in these critically ill patients [8-11]. However, no such clinical study exists to date.

In the present review we first provide a brief history of clinical imaging of the microcirculation and describe how microcirculatory images can be analyzed for measures of microvascular density and perfusion and 
how microcirculatory imaging has been of prognostic value in intensive care patients. Then, we give an overview of therapies potentially improving the microcirculation in critically ill patients (fluid resuscitation, blood transfusion, and vasoactive agents) and propose a clinical trial aimed at demonstrating that therapy targeting improvement of the microcirculation results in improved organ function in patients with severe sepsis and septic shock. Finally, some recent technological advances in clinical microcirculatory image acquisition (image acquisition stabilization) and analysis (automated image analysis) might allow such microcirculationtargeted resuscitation by providing instant feedback on the efficacy of the applied therapeutic strategies at the microcirculatory level.

\section{Brief history of clinical imaging of the microcirculation}

After Van Leeuwenhoek's introduction of in vivo microcirculatory microscopy in 1688 [12,13], this technique was long limited to semi-transparent tissue that could be transilluminated to avoid image contamination by tissue surface reflections and thereby obtain sufficient image contrast [14-16]. Later, use has been made of incident light directed at an oblique angle to the studied tissue [17]. Such a setup, however, required very careful alignment of the light source and the microscopic lens system and still suffered from tissue surface reflections. It was not until 1971 that Sherman and colleagues [18] introduced a new method for studying the microcirculation: incident dark field illumination microscopy. In their setup, dark field illumination was provided through a circular prismatic lens surrounding the objective lens, which created a halo of light around and beyond the objective focal point. This type of illumination gave 'an unusual depth of field and a three-dimensional quality to the tissue observed' and permitted visualization of microcirculatory structures beneath the surface of organs as dark red blood cell columns on a bright background. The authors visualized and photographed the circulation of the cat brain, lung, kidney, liver, mesentery, and intestine successfully.

Freedlander and Lenhart [19] were in 1922 the first to visualize capillaries in living humans and to investigate the effects of infection. In 1987, Slaaf and colleagues [20] developed an alternative way of eliminating tissue surface reflections for imaging subsurface microcirculatory networks that was inspired by fluorescence microscopy. In fluorescence microscopy, image contrast is created by spectral separation of the reflected illumination light and the imaging light by application of an excitation and an emission filter in combination with a dichroic mirror. Similarly, Slaaf and colleagues proposed to separate the reflected illumination light from the imaging light by application of a polarizer and an analyzer (that is, a polarizer oriented orthogonally to the orientation of the polarizer) in combination with a $50 \%$ reflection mirror. Due to its orthogonal orientation with respect to the polarized illumination light, the analyzer blocked directly reflected (undepolarized) light and allowed backscattered (depolarized) light to pass. This setting provided images of the microcirculation at sufficient contrast, similar to those obtained using dark field imaging.

Several years later, Groner and colleagues combined the methods developed by Sherman and colleagues and Slaaf and colleagues and added a spectral component for further optimization of image contrast. In 1999, they introduced orthogonal polarization spectral (OPS) imaging, incorporated into a hand-held, clinically applicable device [21]. Using OPS imaging we were the first to image the human brain microcirculation during surgery [21]. Since then, numerous studies have been undertaken in various clinical scenarios where cardiovascular function is at risk (for example, $[1-3,7,8,10,11]$ ).

Despite the major contribution OPS imaging has made in the field of intravital microcirculatory imaging, several shortcomings were still present $[22,23]$. These include suboptimal imaging of the capillaries due to motioninduced image blurring by movement of the OPS device, the tissue, and/or flowing red blood cells. This introduces difficulties in measuring blood flow velocities in these vessels. Thus, driven by the success of OPS imaging and the drawbacks it has, Goedhart and colleagues [24] have developed a second generation device for clinical imaging of the microcirculation, which was termed sidestream dark field (SDF) imaging. Typical OPS and SDF images obtained at the same sublingual microcirculatory area are presented in Figure 1.

For evaluation of the effects of interventions and (drug) therapy, microcirculatory images can be analyzed to assess (alterations in) microvascular density and perfusion. To assess microcirculatory perfusion, a semiquantitative scoring method (that is, the microcirculatory flow index; MFI) has been developed to characterize microcirculatory flow as 'no flow', 'intermittent flow', 'sluggish flow', and 'continuous flow' [25]. Microcirculatory density can be assessed as the total vessel density (TVD), including perfused and non-perfused microvessels, and perfused vessel density (PVD), including perfused microvessels only. The ratio PVD/TVD is used to express the proportion of perfused vessels (PPV). When only vessels with a diameter $<20 \mu \mathrm{m}$ are included in the analysis, the PVD represents the functional capillary density (FCD), which is considered the main determinant of microcirculatory blood supply.

To date, many studies have investigated the microcirculation using OPS and SDF imaging under various pathophysiological conditions, such as in surgery, 

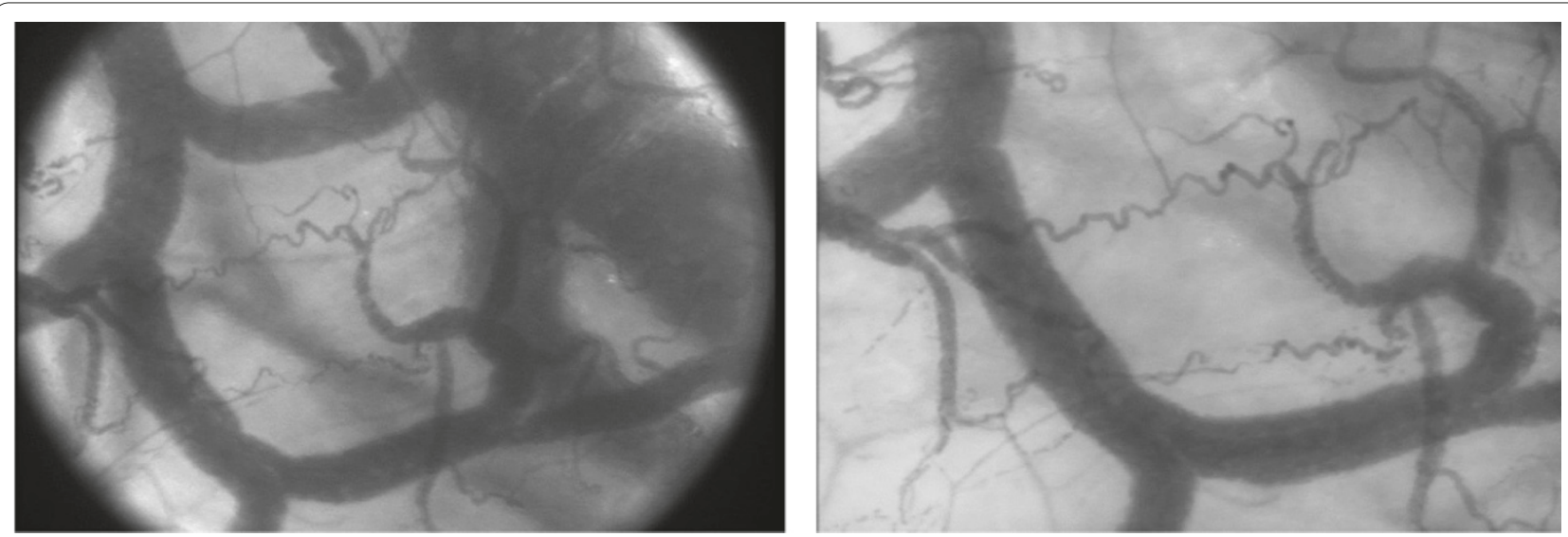

Figure 1. Sublingual microcirculation images obtained at the same microcirculatory area using orthogonal polarization spectral imaging (left) and sidestream dark field imaging (right).

emergency medicine, and intensive care medicine. Both OPS and SDF imaging have had an important clinical impact by observation of the sublingual microcirculation under various pathophysiological conditions and especially during sepsis and shock (for example, [1-3]). Results from several medical centers have shown that alterations in the sublingual microcirculation might provide information with respect to patient outcome from sepsis and shock.

\section{Prognostic value of the microcirculation}

Microcirculatory failure has been shown to be of prognostic value in septic patients. Microcirculatory disorders before resuscitation and their persistence after have been associated with increased risk of morbidity and mortality [1-3,26,27]. De Backer and colleagues [1] found that the microcirculatory alterations in non-surviving septic patients were more severe compared to those in surviving patients. This was later confirmed by Sakr and colleagues and Trzeciak and colleagues, who, furthermore, showed that a lack of improvement of microcirculatory flow after resuscitation was associated with organ failure and death [2] and that non-surviving patients had a significantly higher microcirculatory flow heterogeneity compared to surviving patients [27]. In a later study, Trzeciak and colleagues [3] demonstrated that early increases in microcirculatory perfusion during protocol-directed resuscitation were associated with reduced severity of organ failure as assessed by the Sequential Organ Failure Assessment (SOFA) score in patients with sepsis.

Besides septic patients, microcirculatory disorders have also been shown to predict mortality in patients with acute severe heart failure and cardiogenic shock [28], and impaired microvascular flow was associated with the development of post-operative complications in patients who underwent major abdominal surgery [29].
Hence, a growing body of evidence exists associating depressed microcirculatory function with morbidity and mortality in a wide array of clinical scenarios.

Although many studies have found that microcirculatory dysfunction is a common complication of prognostic value in critically ill patients, most of these studies were single-center investigations only including specific patient populations. To date, therefore, no information on the overall prevalence of microcirculatory dysfunction in intensive care patients is available. To obtain such insight, a large multi-center international observational study has been conducted by Boerma and co-workers to investigate the prevalence of microcirculatory alterations in intensive care patients, regardless of their underlying disease. This is, in fact, the largest microcirculatory study ever performed in the critically ill ( $>400$ patients). Because the study has been designed similarly to the well known multi-center Sepsis Occurrence in Acutely ill Patients (SOAP) studies in which clinical measurements and patient characteristics were recorded at a single time point in many intensive care units throughout the world (for example, [30-33]) but focused on the sublingual microcirculation, it was named the microSOAP study (Microcirculatory Shock Occurrence in Acutely ill Patients registered at ClinicalTrials.gov: NCT01179243). In the microSOAP study, the prevalence of microcirculatory alterations in intensive care patients and the relationship of microcirculatory alterations with the severity of disease in an epidemiological survey were investigated. In one week, the microcirculatory status of all intensive care patients in 40 participating intensive care units worldwide was assessed and patient characteristics were recorded. The patients were followed until death, hospital discharge, or for 60 days. The relationships between microvascular parameters and disease states were analyzed. Once published, this study might provide 
Table 1. Summary of the effects of various interventions on the sublingual microcirculation

\begin{tabular}{|c|c|c|c|c|}
\hline Condition & $\begin{array}{l}\text { Number of } \\
\text { patients }\end{array}$ & Intervention & $\begin{array}{l}\text { Effects on sublingual } \\
\text { microcirculation }\end{array}$ & Reference \\
\hline Septic shock & 8 & Nitroglycerin & Increase in MFI & [25] \\
\hline Severe sepsis or septic shock & 25 & Fluid therapy with saline or HES & $\begin{array}{l}\text { Increase in PVD, PPV, and MFI } \\
\text { Decrease in FHI }\end{array}$ & [34] \\
\hline \multirow[t]{2}{*}{ Severe sepsis } & 37 & $\begin{array}{l}\text { Fluid therapy with RL }(n=16) \text { or HES }(n=21) \\
\text { within } 24 h\end{array}$ & Increase in TVD, PVD, and PPV & [35] \\
\hline & 23 & $\begin{array}{l}\text { Fluid therapy with RL }(n=13) \text { or HES }(n=10) \\
\text { after } 48 \mathrm{~h}\end{array}$ & No changes in TVD, PVD, and PPV & \\
\hline \multirow[t]{3}{*}{ Post-major gastrointestinal surgery } & y 45 & Fluid therapy with RS and CS guided by CVP & Decrease in PVD & [36] \\
\hline & 45 & Fluid therapy with RS and CS guided by SV & No changes in PVD & \\
\hline & 45 & $\begin{array}{l}\text { Fluid therapy with RS and CS guided by } \\
\text { SV + dopexamine }\end{array}$ & Increase in PVD & \\
\hline Severe sepsis & $\begin{array}{c}9 \\
11\end{array}$ & $\begin{array}{l}\text { EGDT with HES } \\
\text { EGDT with saline }\end{array}$ & $\begin{array}{l}\text { At the end of EGDT, PVD, PPV, and MFI were } \\
\text { higher and FHI was lower in HES-treated patients }\end{array}$ & [37] \\
\hline Severe sepsis & 35 & Blood transfusion & $\begin{array}{l}\text { Globally no change in microcirculation, but } \\
\text { negative correlation with baseline perfusion }\end{array}$ & [38] \\
\hline Cardiac surgery & 12 & Blood transfusion & $\begin{array}{l}\text { Increase in TVD and PVD } \\
\text { No changes in MFI }\end{array}$ & [39] \\
\hline Septic shock & 16 & Norepinephrine & No changes in MFI, TVD, PVD, PPV, or FHI & [42] \\
\hline Septic shock & 20 & Norepinephrine & $\begin{array}{l}\text { Globally no change in microcirculation, but } \\
\text { negative correlation with baseline perfusion }\end{array}$ & [43] \\
\hline Septic shock & 22 & $\begin{array}{l}\text { Dobutamine (intravenous; } n=22)+ \\
\text { acetylcholine (topical; } n=10 \text { ) }\end{array}$ & $\begin{array}{l}\text { Dobutamine increased capillary perfusion, but } \\
\text { not capillary density. Acetylcholine completely } \\
\text { restored capillary perfusion }\end{array}$ & [44] \\
\hline Sepsis & $\begin{array}{l}35 \\
35\end{array}$ & $\begin{array}{l}\text { Nitroglycerin } \\
\text { Placebo }\end{array}$ & No differences in MFI between groups & [45] \\
\hline Acute heart failure & 20 & Nitroglycerin & $\begin{array}{l}\text { Increase in PVD, which was reversed after } \\
\text { cessation of nitroglycerin }\end{array}$ & [46] \\
\hline Cardiogenic shock & 19 & Nitroglycerin & Dose-dependent increase in PVD & [47] \\
\hline End-stage chronic heart failure & 8 & & & \\
\hline Severe sepsis & 20 & Activated protein $C$ & $\begin{array}{l}\text { Increase in PPV, which was reversed after } \\
\text { cessation of activated protein C }\end{array}$ & {$[52]$} \\
\hline
\end{tabular}

CS, colloid solution; CVP, central venous pressure; EGDT, early goal-directed therapy; FHI, flow heterogeneity index; HES, hydroxyethyl starch; MFI, microvascular flow index; PPV, proportion of perfused vessels; PVD, perfused vessel density; RL, Ringer's lactate; RS, Ringer's solution; SV, stroke volume; TVD, total vessel density.

valuable information regarding the prevalence of microcirculatory disturbances in intensive care patients and their relationship to the underlying pathophysiology. Furthermore, it is expected that this study will provide a basis for future interventional studies, targeting resuscitation of the microcirculation.

\section{Resuscitation of the microcirculation}

In their key study, Rivers and colleagues [4] have developed an early goal-directed therapeutic protocol in which fluid resuscitation was performed until central venous pressure was 8 to $12 \mathrm{mmHg}$, vasopressor agents were added to maintain the mean arterial pressure above $65 \mathrm{mmHg}$, and red blood cell transfusions and/or inotropic agents were used to increase central venous oxygen saturation to above $70 \%$. With this protocol, Rivers and colleagues significantly reduced the mortality rate in patients with septic shock (31\% versus $47 \%$ for standard therapy). This demonstrates that volume replacement therapy using fluids and/or blood in combination with vasoactive agents is essential for resuscitation of severely septic patients. A summary of the effects of various interventions on the sublingual microcirculation is provided in Table 1.

\section{Fluid resuscitation}

Fluid resuscitation is probably the major therapy aimed at restoring circulating volume and consequently increasing cardiac output and arterial blood pressure in (septic) shock patients. Pottecher and colleagues [34] showed that the sublingual microcirculatory perfusion in severely septic and septic shock patients was significantly improved following fluid loading. As the changes in microcirculation did not correlate to changes in 
macrocirculation, however, the authors suggested that the macro- and microcirculation do not have the same dose-response to fluid loading. This was also observed by Ospina-Tascon and colleagues [35] investigating the response of the macro- and microcirculation to fluid loading in the early (within 24 hours after diagnosis) or late (more than 48 hours after diagnosis) phases of septic shock. The authors found that the microcirculation did increase after fluid loading in the early phase of septic shock but not in the late phase despite significant increases in cardiac output and arterial blood pressure. In patients undergoing major abdominal surgery, Jhanji and colleagues [36] compared stroke volume-guided versus central venous pressure-guided fluid therapy with respect to their effects on microcirculatory perfusion and renal function. The main result was that perfused microvascular density remained normal in the stroke volumeguided therapy group, but decreased in the central venous pressure-guided therapy group. Acute kidney injury was also found more frequently in the central venous pressure-guided therapy group. However, this finding was a post hoc analysis after pooling data from both protocol groups, and other outcome parameters, such as complication rates, mortality, critical care-free days and mortality, were identical in both protocol groups and the control group, despite the improvement in microcirculation.

Hence, these studies indicate that fluid loading is an effective first step in the resuscitation of the microcirculation. In addition, Dubin and colleagues [37] demonstrated in a randomized controlled study in septic patients that a $6 \% \mathrm{HES} / 0.4$ solution had superior microcirculatory recruitment power compared to a saline solution in early goal-directed therapy. In this study, however, baseline microcirculation was not assessed, making it difficult to understand whether differences at 24 hours result from differences at baseline or from specific effects of different types of fluids. Moreover, no outcome data are yet available showing benefit from synthetic colloids over crystalloids.

\section{Blood transfusion}

Both OPS and SDF imaging have been used to investigate the direct effects of red blood cell (RBC) transfusions on the microcirculation [38,39]. Sakr and colleagues [38] studied sublingual microcirculation in 35 septic patients using orthogonal polarization spectral imaging. They performed the measurements just before RBC unit transfusion and one hour after transfusion of one or two leukoreduced RBC units with a mean age of 24 days. They found that although mean arterial pressure and oxygen delivery increased following RBC transfusion, oxygen uptake and microcirculatory parameters did not. It must be noted, however, that there was interindividual variability with an increase in sublingual capillary perfusion in patients with depressed perfusion at baseline and a decrease in perfusion in patients with normal baseline perfusion [38]. In contrast, our group has demonstrated an increased sublingual microcirculatory density and tissue oxygenation after transfusion of one to three RBC units with a mean age of 18 days in cardiac surgery patients [39]. In this study we were able to verify that the transfused blood is effective in improving oxygen transport to the tissue by promoting $\mathrm{RBC}$ delivery to the microcirculation and identified the mechanism by which this is accomplished: that is, not by increasing microcirculatory flow velocity but rather by filling empty capillaries, thereby reducing the oxygen diffusion distances to the tissue cells. However, whether this leads to improved oxygen consumption remains to be investigated. Parallel to the findings by Sakr and colleagues, we have recently conducted a pilot study to investigate the efficacy of RBC transfusions to improve microcirculatory density in adult septic patients and also found no improvement in the microcirculation after blood transfusion in these patients [40]. A potential explanation for this is that, in sepsis, hemorheological alterations and damaged host microcirculation (for example, endothelium and glycocalyx) could diminish the efficacy of RBC transfusions to correct anemia at the microcirculatory level. However, this warrants further study.

\section{Vasoactive agents}

Vasoactive agents such as norepinephrine, epinephrine, dopamine, dopexamine, and dobutamine are often used in hypotensive (septic) shock patients to increase blood pressure and restore the systemic hemodynamic state. These agents also have an impact on the microcirculation, as reviewed by Boerma and Ince [41]. The general finding is that while being effective at increasing blood pressure, vasopressors can have various effects on the microcirculation. Jhanji and colleagues [42] found in septic shock patients that norepinepherine, while increasing blood pressure, was completely ineffective at promoting microcirculatory blood flow. In another study by Jhanji and colleagues [36] it was found that a treatment algorithm incorporating stroke volume-guided fluid therapy and a low-dose dopexamine infusion increased global oxygen delivery and central venous oxygen saturation in association with significant improvements in sublingual and cutaneous microvascular flow, while stroke volumeguided fluid therapy alone was associated with more modest improvements in global hemodynamics and microvascular flow. In a similar study, Dubin and colleagues [43] found that norepinephrine in hypotensive patients with low microcirculation was able to increase microvascular flow, but in equally hypotensive patients 
with a normal microcirculation norepinephrine actually decreased microvascular flow. These studies emphasize that using a fixed target of blood pressure alone to guide resuscitation does not guarantee improvement of the microcirculation. Although in an earlier study De Backer and colleagues had showed that the proportion of perfused vessels was similar in patients treated with or without adrenergic agents [1], they later showed in septic shock patients that dobutamine infusion $(5 \mu \mathrm{gg} / \mathrm{kg} /$ minute) markedly reduced the proportion of non-perfused capillaries [44]. The authors furthermore showed in a subset of patients that topical application of acetylcholine could further improve microcirculatory perfusion, which suggests that the dobutamine infusion, although recruiting some capillaries, did not fully open the microcirculation.

As mentioned above, the vasodilatory action of acetylcholine was able to recruit the capillaries of the sublingual microcirculation in patients with severe sepsis [44]. In line with this, Spronk and colleagues [25] found that intravenous infusion of nitroglycerin improved microcirculatory perfusion in septic shock patients. In a placebo-controlled randomized trial in septic patients, however, Boerma and colleagues [45] did not find such beneficial effects of intravenous infusion of nitroglycerin after fulfillment of protocol-driven resuscitation endpoints. The authors showed an equal change in microcirculatory flow in all groups over the first 24 hours of intensive care with no significant effects of nitroglycerin. During cardiogenic shock, in contrast, Den Uil and colleagues $[46,47]$ found that nitroglycerin improved the sublingual microcirculation in a dose-dependent fashion. Interestingly, the observed improvement of the microcirculation was not correlated with changes in cardiac output or arterial blood pressure and disappeared after cessation of nitroglycerin infusion. Alternative routes for nitric oxide administration (for example, inhaled nitric oxide) are being explored to improve the microcirculation without worsening the macrocirculation, as extensively discussed by Trzeciak and colleagues [9].

Another agent with potential for improving microvascular function in critically ill patients is recombinant activated protein $\mathrm{C}$ (APC), which decreases the uncontrolled cascades of inflammation and coagulation and impaired fibrinolysis in sepsis $[48,49]$. Bernard and colleagues [50] have shown that exogenous APC administration significantly reduced organ failure and improved survival in severely septic patients, although this was later questioned by Silva and colleagues [51]. De Backer and colleagues [52] reported that severely septic patients had an increased proportion of perfused microvessels while receiving continuous infusion of APC. Once APC infusion stopped, microvascular perfusion transiently decreased. The authors furthermore showed that the improved microvascular perfusion was associated with more rapid resolution of hyperlactatemia.

\section{Targeting the microcirculation}

Even after interventions effectively optimizing macrocirculatory hemodynamics, high mortality rates still persist in critically ill and especially in septic patients. Therefore, rather than limiting therapy to macrocirculatory targets alone, microcirculatory targets could be incorporated to potentially reduce mortality rates in these critically ill patients [8-11]. Although an association between an abnormal microcirculation and adverse outcome may be confirmed world-wide, this does not imply that improving the microcirculation in these conditions will improve outcome of these patients. A randomized study should be conducted to prove that using microcirculatory parameters as end-points of resuscitation indeed improves outcome of the patients. However, no such clinical study yet exists.

Such a trial would, for the first time, implement a resuscitation strategy based on resolving microcirculatory disorders known to be associated with increased morbidity and mortality in the intensive care unit. This novel goal-directed therapeutic strategy might, if successful, have a large impact on the care of intensive care patients. If not (or less) successful, this could be due either to the wrong choice of drug or to the secondary rather than primary role of microcirculatory failure in morbidity and mortality in the critically ill. With such a trial, microcirculatory diagnostics will be taken to the next level where the microcirculation will be used as a therapeutic target in the treatment of septic patients.

\section{Recent technological advances} Image acquisition stabilization

Optimizing microcirculatory density and perfusion has become the focus of new clinical studies and microcirculatory images are therefore gaining a more prominent role in clinical research. Proper interpretation of microcirculatory images is essential and relies on the quality of the images with respect to axial and lateral stability. Since both OPS and SDF imaging technologies are incorporated into hand-held microscopes, operational issues may arise in terms of axial and lateral instability of the microscope probes, potentially causing pressure artifacts and image drifting, respectively. The current guidelines for microcirculatory image acquisition dictate that three to five microcirculatory sites should be measured per time point with a minimal recording time of $20 \mathrm{~s}$ per site to allow reliable analysis of microcirculatory density and perfusion [53]. Image drifting, however, makes this particularly difficult in both sedated and awake patients. Pressure artifacts, in addition, can alter mucosal capillary blood flow, thereby limiting the 
use of the captured images for determination of microcirculatory perfusion.

To improve microcirculatory image acquisition, Balestra and colleagues [54] have developed, evaluated, and validated an image acquisition stabilizer for the SDF imaging device. The stabilizer was based on application of negative pressure to the periphery of the microscopic field of view to create adherence of the microscope probe to the tissue of interest. The authors found that the stabilizer did not affect microcirculatory perfusion in the SDF imaging field of view and prevented pressure artifacts up to a significantly greater force applied by the SDF probe onto the tissue. Furthermore, the duration of maintaining a stable image sequence was significantly increased with the stabilizer $(8 \pm 2 \mathrm{~s}$ without versus $42 \pm 8 \mathrm{~s}$ with the stabilizer). Ultimately, the authors described that, using the stabilizer and a mechanical arm, it was possible to perform microcirculatory measurements without the need for an operator. Hence, instead of multiple measurements to determine the microcirculatory state at a certain time point, continuous measurements of microcirculatory perfusion and density could be made during a clinical maneuver or intervention.

\section{Rapid automated image analysis}

For evaluation of the effects of interventions and (drug) therapy, SDF images are analyzed to assess (alterations in) microvascular density and perfusion. To reduce the time required for SDF image analysis for microvascular density and perfusion, Dobbe and colleagues [55] have developed and validated a method that has been commercialized into a software package termed Automated Vascular Analysis. However, the semi-automatic offline analysis of the SDF images is still a time consuming endeavor requiring a significant amount of user interaction. This severely limits the bedside use of SDF imaging as a diagnostic tool.

Our group has recently developed a rapid and fully automatic method for the assessment of microvascular density and perfusion in SDF images [56]. We improved the algorithms for microvascular density assessment incorporated in the Automated Vascular Analysis software and introduced a new method for microvascular perfusion assessment. We showed that the new method was very rapid ( $<30 \mathrm{~s}$ per clip) and adequately recovered total vessel density. With video simulations, we showed that the detection of perfusion using the new method was possible, but was limited at high cell densities and velocities at a $25 \mathrm{~Hz}$ imaging rate. In high quality SDF video clips, however, the new method was able to discriminate between perfused and non-perfused microvasculature. With video simulations it was furthermore shown that the limitations of the new method were mainly hardware-related and could be overcome by implementing more advanced camera technology in SDF imaging (that is, higher spatial and temporal resolution).

For future SDF imaging research, the automatic microvascular density assessment can be combined with manually assigning a flow score to each quadrant of the image as proposed by Spronk and colleagues [25], evaluated by others $[57,58]$, and included in the standard operating procedures as dictated by a consensus on microcirculatory image acquisition and analysis [53]. Although this introduces some user interaction, it allows analysis of microvascular density and perfusion in SDF video clips within a few minutes and may allow assessment of microcirculation at the bedside.

\section{Novel video microscopy technology}

As described above, current OPS and SDF imaging devices can be regarded as first and second generation devices, respectively, employing relatively low resolution analogue camera technology. Braedius Scientific is currently in the process of introducing a potential third generation device as an improved imaging modality for more comprehensive clinical observation of the microcirculation. A computer-controlled digital camera incorporated in the device will have a much higher spatial (14 megapixels versus 1.3 megapixels) and temporal (60 versus 25 frames per second) resolution as well as shorter camera exposure times compared to the previous generation devices. This device, with increased spatial and temporal resolution in combination with a sensor attached to a powerful computer, might provide the needed hardware requirements to allow instant online analysis of microcirculatory images needed at the bedside for clinical decision making for guidance of microcirculatory-targeted therapies.

\section{Conclusion}

A growing body of evidence exists underlining that depressed microcirculatory function is associated with morbidity and mortality in a wide array of clinical scenarios and that even after interventions effectively optimizing macrocirculatory hemodynamics, high mortality rates still persist in critically ill and especially in septic patients. Therefore, rather than limiting therapy to macrocirculatory targets alone, microcirculatory targets could be incorporated to potentially reduce mortality rates in these critically ill patients. To date, no such clinical study yet exists due to the unavailability of bedside technology scoring microvascular density and perfusion in real time. However, recent technological advances in the field of microcirculatory image acquisition and analysis might allow such microcirculationtargeted resuscitation by providing instant feedback on the efficacy of the applied therapeutic strategies at the microcirculatory level. 


\section{Abbreviations}

APC, activated protein C; OPS, orthogonal polarization spectral; PPV, proportion of perfused vessels; PVD, perfused vessel density; RBC, red blood cell; SDF, side stream dark field; TVD, total vessel density.

\section{Competing interests}

$\mathrm{Cl}$ is the inventor of SDF technology that is commercialized by MicroVision Medical. He has been a consultant for this company in the past, but he has broken all contact with this company for more than two years now. $\mathrm{Cl}$ also has no competing interests in MicroVision Medical, Cytometrics, or Braedius Scientific other than his commitment to promote the importance of microcirculation in the care of critically ill patients.

\section{Author details}

'Department of Intensive Care, Erasmus MC, University Medical Center Rotterdam, 's-Gravendijkwal 230, 3015 CE Rotterdam, the Netherlands. ${ }^{2}$ Department of Translational Physiology, Academic Medical Center, University of Amsterdam, Meibergdreef 9,1105 AZ Amsterdam, the Netherlands. ${ }^{3}$ Department of Intensive Care, Medical Center Alkmaar, Wilhelminalaan 12 1815 JD Alkmaar, the Netherlands.

Published: 19 June 2012

\section{References}

1. De Backer D, Creteur J, Preiser JC, Dubois MJ, Vincent JL: Microvascular blood flow is altered in patients with sepsis. Am J Respir Crit Care Med 2002, 166:98-104.

2. Sakr Y, Dubois MJ, De Backer D, Creteur J, Vincent JL: Persistent microcirculatory alterations are associated with organ failure and death in patients with septic shock. Crit Care Med 2004, 32:1825-1831.

3. Trzeciak S, McCoy JV, Phillip Dellinger R, Arnold RC, Rizzuto M, Abate NL, Shapiro NI, Parrillo JE, Hollenberg SM; Microcirculatory Alterations in Resuscitation and Shock (MARS) investigators: Early increases in microcirculatory perfusion during protocol-directed resuscitation are associated with reduced multi-organ failure at $24 \mathrm{~h}$ in patients with sepsis. Intensive Care Med 2008, 34:2210-2217.

4. Rivers E, Nguyen B, Havstad S, Ressler J, Muzzin A, Knoblich B, Peterson E, Tomlanovich M; Early Goal-Directed Therapy Collaborative Group: Early goaldirected therapy in the treatment of severe sepsis and septic shock. N Engl J Med 2001, 345:1368-1377.

5. Lin SM, Huang CD, Lin HC, Liu CY, Wang CH, Kuo HP: A modified goaldirected protocol improves clinical outcomes in intensive care unit patients with septic shock: a randomized controlled trial. Shock 2006 26:551-557

6. Otero RM, Nguyen HB, Huang DT, Gaieski DF, Goyal M, Gunnerson KJ, Trzeciak S, Sherwin R, Holthaus CV, Osborn T, Rivers EP: Early goal-directed therapy in severe sepsis and septic shock revisited: concepts, controversies, and contemporary findings. Chest 2006, 130:1579-1595.

7. Ince C: The microcirculation is the motor of sepsis. Crit Care 2005 9 Suppl 4:S13-19.

8. Bateman RM, Walley KR: Microvascular resuscitation as a therapeutic goal in severe sepsis. Crit Care 2005, 9(Suppl 4):S27-S32.

9. Trzeciak S, Cinel I, Phillip Dellinger R, Shapiro NI, Arnold RC, Parrillo JE, Hollenberg SM; Microcirculatory Alterations in Resuscitation and Shock (MARS) Investigators: Resuscitating the microcirculation in sepsis: the central role of nitric oxide, emerging concepts for novel therapies, and challenges for clinical trials. Acad Emerg Med 2008, 15:399-413.

10. Nencioni A, Trzeciak S, Shapiro NI: The microcirculation as a diagnostic and therapeutic target in sepsis. Intern Emerg Med 2009, 4:413-418.

11. Harrois A, Dupic L, Duranteau J: Targeting the microcirculation in resuscitation of acutely unwell patients. Curr Opin Crit Care 2011, 17:303-307.

12. Van Leeuwenhoek A: Letter 65. Read at the Royal Society; 1688

13. Dobell C: Antony van Leeuwenhoek and His "Little Animals". Harcourt, Brace and Company: New York; 1932

14. Hall HL: A study of the pulmonary circulation by the transillumination method. Am J Physiol 1925, 72:446.

15. Irwin JW, Burrage WS, Aimar CE, Chesnut RW Jr: Microscopical observations of the pulmonary arterioles, capillaries, and venules of living guinea pigs and rabbits. Anat Rec 1954, 119:391-407.

16. Krahl VE: Observations on the pulmonary alveolus and its capillary circulation in the living rabbit. Anat Rec 1962, 142:350.

17. Krahl VE: In vivo microscopy of the rabbit's lung. Bibl Anat Fasc 1964, 4:400.

18. Sherman H, Klausner S, Cook WA: Incident dark-field illumination: a new method for microcirculatory study. Angiology 1971, 22:295-303.

19. Freedlander $\mathrm{SO}$, Lenhart $\mathrm{CH}$ : Clinical observations on the capillary circulation. Arch Intern Med 1922, 29:12-32

20. Slaaf DW, Tangelder GJ, Reneman RS, Jäger K, Bollinger A: A versatile incident illuminator for intravital microscopy. Int J Microcirc Clin Exp 1987, 6:391-397.

21. Groner W, Winkelman JW, Harris AG, Ince C, Bouma GJ, Messmer K, Nadeau RG: Orthogonal polarization spectral imaging: a new method for study of the microcirculation. Nat Med 1999, 5:1209-1212.

22. Lindert J, Werner J, Redlin M, Kuppe $H$, Habazettl $H$, Pries AR: OPS imaging of human microcirculation: a short technical report. J Vasc Res 2002 39:368-372.

23. Cerný V, Turek Z, Parízková R: Orthogonal polarization spectral imaging Physiol Res 2007, 56:141-147.

24. Goedhart PT, Khalilzada M, Bezemer R, Merza J, Ince C: Sidestream Dark Field (SDF) imaging: a novel stroboscopic LED ring-based imaging modality for clinical assessment of the microcirculation. Opt Express 2007, 15:15101-15114

25. Spronk PE, Ince C, Gardien MJ, Mathura KR, Oudemans-van Straaten HM Zandstra DF: Nitroglycerin in septic shock after intravascular volume resuscitation. Lancet 2002, 360:1395-1396.

26. Vincent $\mathrm{J}$, De Backer D: Microvascular dysfunction as a cause of organ dysfunction in severe sepsis. Crit Care 2005, 9(Suppl 4):S9-S12

27. Trzeciak S, Dellinger RP, Parrillo JE, Guglielmi M, Bajaj J, Abate NL, Arnold RC, Colilla S, Zanotti S, Hollenberg SM; Microcirculatory Alterations in Resuscitation and Shock Investigators: Early microcirculatory perfusion derangements in patients with severe sepsis and septic shock: relationship to hemodynamics, oxygen transport, and survival. Ann Emerg Med 2007, 49:88-98.

28. De Backer D, Creteur J, Dubois MJ, Sakr Y, Vincent JL: Microvascular alterations in patients with acute severe heart failure and cardiogenic shock. Am Heart J 2004, 147:91-99.

29. Jhanji S, Lee C, Watson D, Hinds C, Pearse RM: Microvascular flow and tissue oxygenation after major abdominal surgery: association with postoperative complications. Intensive Care Med 2009, 35:671-677.

30. Payen D, de Pont AC, Sakr Y, Spies C, Reinhart K, Vincent JL; Sepsis Occurrence in Acutely III Patients (SOAP) Investigators: A positive fluid balance is associated with a worse outcome in patients with acute renal failure. Crit Care 2008, 12:R74

31. Sakr Y, Reinhart K, Vincent JL, Sprung CL, Moreno R, Ranieri VM, De Backer D, Payen D: Does dopamine administration in shock influence outcome? Results of the Sepsis Occurrence in Acutely III Patients (SOAP) Study. Crit Care Med 2006, 34:589-597.

32. Sprung CL, Sakr Y, Vincent JL, Le Gall JR, Reinhart K, Ranieri VM, Gerlach H, Fielden J, Groba CB, Payen D: An evaluation of systemic inflammatory response syndrome signs in the Sepsis Occurrence In Acutely III Patients (SOAP) study. Intensive Care Med 2006, 32:421-427.

33. Vincent JL, Sakr Y, Sprung C, Harboe S, Damas P; Sepsis Occurrence in Acutely Ill Patients (SOAP) Investigators: Are blood transfusions associated with greater mortality rates? Results of the Sepsis Occurrence in Acutely III Patients study. Anesthesiology 2008, 108:31-39.

34. Pottecher J, Deruddre S, Teboul JL, Georger JF, Laplace C, Benhamou D, Vicaut E, Duranteau J: Both passive leg raising and intravascular volume expansion improve sublingual microcirculatory perfusion in severe sepsis and septic shock patients. Intensive Care Med 2010, 36:1867-1874.

35. Ospina-Tascon G, Neves AP, Occhipinti G, Donadello K, Büchele G, Simion D, Chierego ML, Silva TO, Fonseca A, Vincent JL, De Backer D: Effects of fluids on microvascular perfusion in patients with severe sepsis. Intensive Care Med 2010, 36:949-955

36. Jhanji S, Vivian-Smith A, Lucena-Amaro S, Watson D, Hinds CI, Pearse RM: Haemodynamic optimisation improves tissue microvascular flow and oxygenation after major surgery: a randomised controlled trial. Crit Care 2010, 14:R151

37. Dubin A, Pozo MO, Casabella CA, Murias G, Pálizas F Jr, Moseinco MC, Kanoore Edul VS, Pálizas F, Estenssoro E, Ince C: Comparison of $6 \%$ hydroxyethyl starch 130/0.4 and saline solution for resuscitation of the microcirculation during the early goal-directed therapy of septic patients. J Crit Care 2010 , 25:659.

38. Sakr Y, Chierego M, Piagnerelli M, Verdant C, Dubois MJ, Koch M, Creteur J, 
Gullo A, Vincent JL, De Backer D: Microvascular response to red blood cell transfusion in patients with severe sepsis. Crit Care Med 2007, 35:1639-1644.

39. Yuruk K, Almac E, Bezemer R, Goedhart P, de Mol B, Ince C: Blood transfusions recruit the microcirculation during cardiac surgery. Transfusion 2011, 51:961-967.

40. Ayhan B, Yuruk K, Bakker J, de Mol BAJM, Ince C: Blood transfusions recruit the microcirculation in on-pump cardiac surgery patients, but not in septic patients. Intensive Care Med 2010, 36(Suppl S208):0489.

41. Boerma $E C$, Ince $C$ : The role of vasoactive agents in the resuscitation of microvascular perfusion and tissue oxygenation in critically ill patients. Intensive Care Med 2010, 36:2004-2018.

42. Jhanii S, Stirling S, Patel N, Hinds CJ, Pearse RM: The effect of increasing doses of norepinephrine on tissue oxygenation and microvascular flow in patients with septic shock. Crit Care Med 2009, 37:1961-1966.

43. Dubin A, Pozo MO, Casabella CA, Pálizas F Jr, Murias G, Moseinco MC, Kanoore Edul VS, Pálizas F, Estenssoro E, Ince C: Increasing arterial blood pressure with norepinephrine does not improve microcirculatory blood flow: a prospective study. Crit Care 2009, 13:R92.

44. De Backer D, Creteur J, Dubois MJ, Sakr Y, Koch M, Verdant C, Vincent JL: The effects of dobutamine on microcirculatory alterations in patients with septic shock are independent of its systemic effects. Crit Care Med 2006 34:403-408.

45. Boerma EC, Koopmans M, Konijn A, Kaiferova K, Bakker AJ, van Roon EN, Buter H, Bruins N, Egbers PH, Gerritsen RT, Koetsier PM, Kingma WP, Kuiper MA, Ince C: Effects of nitroglycerin on sublingual microcirculatory blood flow in patients with severe sepsis/septic shock after a strict resuscitation protocol: a double-blind randomized placebo controlled trial. Crit Care Med 2010, 38:93-100.

46. den Uil CA, Lagrand WK, Spronk PE, van der Ent M, Jewbali LS, Brugts JJ, Ince C, Simoons ML: Low-dose nitroglycerin improves microcirculation in hospitalized patients with acute heart failure. Eur J Heart Fail 2009, 11:386-390.

47. den Uil CA, Caliskan K, Lagrand WK, van der Ent M, Jewbali LS, van Kuijk JP, Spronk PE, Simoons ML: Dose-dependent benefit of nitroglycerin on microcirculation of patients with severe heart failure. Intensive Care Med 2009, 35:1893-1899.

48. Levi M, van der Poll T, ten Cate H, van Deventer SJ: The cytokine-mediated imbalance between coagulant and anticoagulant mechanisms in sepsis and endotoxaemia. Eur J Clin Invest 1997, 27:3-9.
49. Joyce DE, Nelson DR, Grinnell BW: Leukocyte and endothelial cell interactions in sepsis: relevance of the protein $\mathrm{C}$ pathway. Crit Care Med 2004, 32 (5 Suppl):S280-S286.

50. Bernard GR, Vincent JL, Laterre PF, LaRosa SP, Dhainaut JF, Lopez-Rodriguez A, Steingrub JS, Garber GE, Helterbrand JD, Ely EW, Fisher CJ Jr; Recombinant human protein C Worldwide Evaluation in Severe Sepsis (PROWESS) study group: Efficacy and safety of recombinant human activated protein C for severe sepsis. N Eng/ J Med 2001, 344:699-709.

51. Silva E, de Fiqueiredo LF, Colombari F: Colombari F. Prowess-shock trial: a protocol overview and perspectives. Shock 2010, 34 (Suppl 1):48-53.

52. De Backer D, Verdant C, Chierego M, Koch M, Gullo A, Vincent JL: Effects of drotrecogin alfa activated on microcirculatory alterations in patients with severe sepsis. Crit Care Med 2006, 34:1918-1924.

53. De Backer D, Hollenberg S, Boerma C, Goedhart P, Büchele G, Ospina-Tascon G, Dobbe I, Ince C: How to evaluate the microcirculation: report of a round table conference. Crit Care 2007, 11:R101.

54. Balestra GM, Bezemer R, Boerma EC, Yong ZY, Sjauw KD, Engstrom AE, Koopmans M, Ince C: Improvement of sidestream dark field imaging with an image acquisition stabilizer. BMC Med Imaging 2010, 10:15.

55. Dobbe JG, Streekstra GJ, Atasever B, van Zijderveld R, Ince C: Measurement of functional microcirculatory geometry and velocity distributions using automated image analysis. Med Biol Eng Comput 2008, 46:659-670.

56. Bezemer R, Dobbe JG, Bartels SA, Christiaan Boerma E, Elbers PW, Heger M, Ince $C$ : Rapid automatic assessment of microvascular density in sidestream dark field images. Med Biol Eng Comput, 49:1269-1278.

57. Dubin A, Pozo MO, Ferrara G, Murias G, Martins E, Canullán C, Canales HS, Kanoore Edul VS, Estenssoro E, Ince C: Systemic and microcirculatory responses to progressive hemorrhage. Intensive Care Med 2009, 35:556-564

58. Arnold RC, Parrillo JE, Phillip Dellinger R, Chansky ME, Shapiro NI, Lundy DJ, Trzeciak S, Hollenberg SM: Point-of-care assessment of microvascular blood flow in critically ill patients. Intensive Care Med 2009, 35:1761-1766.

doi:10.1186/cc11236

Cite this article as: Bezemer $R$, et al: Clinical review: Clinical imaging of the sublingual microcirculation in the critically ill - where do we stand? Critical Care 2012, 16:224 